\title{
Supraconvergent cell-centered scheme for two dimensional elliptic problems
}

\author{
S. Barbeiro \\ CMUC, Department of Mathematics, University of Coimbra, Apartado 3008, 3001-454 Coimbra, Portugal
}

\begin{abstract}
In this paper we study the convergence properties of a cell-centered finite difference scheme for second order elliptic equations with variable coefficients subject to Dirichlet boundary conditions. We prove that the finite difference scheme on nonuniform meshes although not even being consistent are nevertheless second order convergent. More precisely, second order convergence with respect to a discrete version of $L^{2}(\Omega)$-norm is shown provided that the exact solution is in $H^{4}(\Omega)$. Estimates for the difference between the pointwise restriction of the exact solution on the discretization nodes and the finite difference solution are proved. The convergence is studied with the aid of an appropriate negative norm. A numerical example support the convergence result.
\end{abstract}

(C) 2007 Published by Elsevier B.V. on behalf of IMACS.

Keywords: Cell-centered scheme; Nonuniform mesh; Supraconvergence

\section{Introduction}

In the last decades there has been a strong mathematical interest in numerical discretization methods that have higher convergence order than expected by analyzing the truncation error in a standard way. In the context of finite difference schemes on nonequidistant grids this behavior is called supraconvergence. Different methods of proving supraconvergence of finite difference schemes for ordinary differential equations have been used by the various authors (see e.g. $[3,6,12,15,18,19,24]$ and [27]). The phenomenon of supraconvergence in more than one space dimension has also been studied in the literature (see e.g. [7,10,11] and [21]). The topic in the context of finite element methods has been treated in the papers $[3,4,10,13,16,17,20,22,23,25,33]$.

We are interested in studying this phenomenon in a variant of finite differences, the so called cell-centered schemes, which are used in many codes. In fact, these schemes are not even consistent but nevertheless second order convergent. This fact is noticed by Tikhonov and Samarskii in [31]. Russell and Wheeler [26] use the equivalence of a cellcentered finite difference method and a mixed finite element method with a special quadrature formula for proving first order convergence of the solution and its gradient. Manteuffel and White [24] show second order convergence in both vertex-centered finite difference schemes and cell-centered finite difference schemes for scalar problems, on nonuniform meshes. Supraconvergence results for a two-dimensional cell-centered scheme are presented by Forsyth and Sammon [11] and also by Weiser and Wheeler [32], among others.

E-mail address: silvia@mat.uc.pt.

URL: http://www.mat.uc.pt/ silvia. 
Our main purpose is to analyze how supraconvergence come out for more general problems than considered so far. The analysis of the present paper is based on using negative norms. The analysis of supraconvergence with one additional order of convergence in [3] and [7] is more or less explicitly based on the concept of negative norms. In these two papers discrete analogues of the $H^{-1}$-norm were considered. The concept of negative norms in the analysis of supraconvergence was also used in $[4,7-10,15]$ and [17]. The idea in the present paper is to work instead with a discrete version of the $H^{-2}$-norm. The convergence result relies on a stability inequality with respect to this norm. We consider the error as the difference between the pointwise restriction of the solution on the discretization nodes and the finite difference solution. Error estimates of order 2, in a discrete version of the $L^{2}(\Omega)$-norm, are proved if the exact solution is in the Sobolev space $H^{4}(\Omega)$.

The analysis of supraconvergence with two additional orders of convergence for the one-dimensional case is considered in [2], with the aid of so-called Spijker norms [28] which are defined using certain summation operators. These operators are applied twice corresponding to the two gained additional orders of convergence. The use of Spijker norms is restricted to one dimension but they give the idea for a generalization to higher dimensions because they are related to the negative norms (for more details see [2]). In the present paper we use this kind of analysis for two dimensional problems.

We consider the discretization of the following elliptic differential equation

$$
A u:=-\left(a u_{x}\right)_{x}-\left(c u_{y}\right)_{y}+d u_{x}+e u_{y}+f u=g \quad \text { on } \Omega,
$$

subject to the Dirichlet boundary condition

$$
u=\psi \quad \text { in } \partial \Omega .
$$

The coefficients of $A$ are assumed to satisfy $a, c \in W^{3, \infty}(\Omega), d, e, f \in W^{2, \infty}(\Omega)$ and $a(x, y) \geqslant \underline{a}>0, c(x, y) \geqslant$ $\underline{c}>0, \forall(x, y) \in \Omega$. We also assume $\psi \in H^{1 / 2}(\partial \Omega)$. The domain $\Omega$ is a union of rectangles.

In order to prepare the definition of the cell-centered finite difference approximation of (1)-(2) let us first introduce the nonuniform grid $G_{H}$. Let $x_{-1}, x_{N+1}, y_{-1}$ and $y_{M+1}$ be the vertices of a rectangle $R=\left(x_{-1}, x_{N+1}\right) \times\left(y_{-1}, y_{M+1}\right)$ which contains $\Omega$. We define the grid $G_{H}:=R_{1} \times R_{2}$, where

$$
R_{1}:=\left\{x_{-1}<x_{0}<\cdots<x_{N}<x_{N+1}\right\}, \quad R_{2}:=\left\{y_{-1}<y_{0}<\cdots<y_{M}<y_{M+1}\right\} .
$$

The grid $G_{H}$ is assumed to satisfy the following condition: the vertices of $\Omega$ are centers of the rectangles formed by $G_{H}$. Let

$$
S_{H}:=\left\{\left(x_{j-1 / 2}, y_{\ell-1 / 2}\right): j=0, \ldots, N+1, \ell=0, \ldots, M+1\right\},
$$

where $x_{j-1 / 2}:=\left(x_{j-1}+x_{j}\right) / 2, y_{\ell-1 / 2}:=\left(y_{\ell-1}+y_{\ell}\right) / 2$. Our aim is to obtain numerical solutions in $\Omega_{H}:=S_{H} \cap \Omega$. We define also $\partial \Omega_{H}:=S_{H} \cap \partial \Omega$ and $\bar{\Omega}_{H}:=\Omega_{H} \cup \partial \Omega_{H}$.

Fig. 1 illustrates the cell-centered grid in the domain.

In the case of a rectangular domain $\Omega=\left(x_{0}, x_{N}\right) \times\left(y_{0}, y_{M}\right)$, we allow both $R=\Omega$ and $R \supset \Omega$, i.e. we consider $x_{-1} \leqslant x_{0}, x_{N} \leqslant x_{N+1}, y_{-1} \leqslant y_{0}$ and $y_{M} \leqslant y_{M+1}$.

For the formulation of the difference problem we use the centered divided difference in $x$-direction

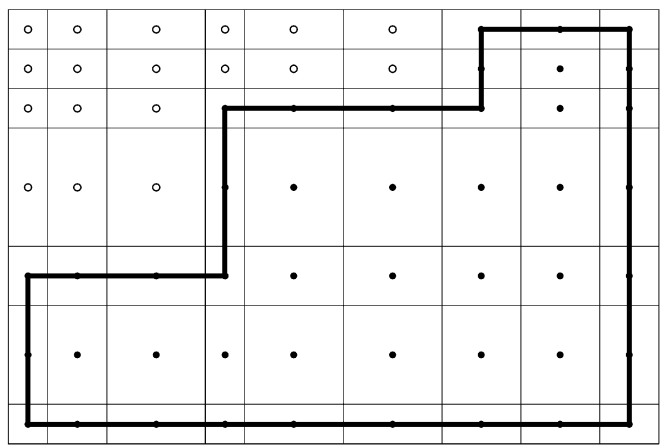

$\circ \in \underline{S}_{H} \backslash \bar{\Omega}_{H}$

$\cdot \in \bar{\Omega}_{H}$

Fig. 1. Domain and grid points. 


$$
\begin{aligned}
& \left(\delta_{x} v_{H}\right)_{j, \ell+1 / 2}:=\frac{v_{j+1 / 2, \ell+1 / 2}-v_{j-1 / 2, \ell+1 / 2}}{h_{j-1 / 2}}, \\
& \left(\delta_{x} w_{H}\right)_{j-1 / 2, \ell+1 / 2}:=\frac{w_{j, \ell+1 / 2}-w_{j-1, \ell+1 / 2}}{h_{j-1}},
\end{aligned}
$$

where $h_{j-1 / 2}:=x_{j+1 / 2}-x_{j-1 / 2}, h_{j-1}:=x_{j}-x_{j-1}$. Correspondingly, the divided difference with respect to the $y$ variable are defined, with the mesh-size vector $k$ in place of $h$. The difference problem is to find the solution $u_{H}$ such that

$$
\begin{aligned}
& A_{H} u_{H}=M_{H} R_{G_{H}} g \quad \text { in } \Omega_{H}, \\
& u_{H}=R_{H} \psi \quad \text { on } \partial \Omega_{H},
\end{aligned}
$$

where the difference operator $A_{H}$ is given by

$$
A_{H} u_{H}:=-\delta_{x}\left(a \delta_{x} u_{H}\right)-\delta_{y}\left(c \delta_{y} u_{H}\right)+M_{x}\left(d \delta_{x} u_{H}\right)+M_{y}\left(e \delta_{y} u_{H}\right)+f u_{H},
$$

and

$$
\begin{aligned}
\left(M_{x} w_{H}\right)_{j-1 / 2, \ell-1 / 2} & :=\frac{w_{j-1, \ell-1 / 2}+w_{j, \ell-1 / 2}}{2}, \\
\left(M_{y} w_{H}\right)_{j-1 / 2, \ell-1 / 2} & :=\frac{w_{j-1 / 2, \ell-1}+w_{j-1 / 2, \ell}}{2}, \\
\left(M_{H} w_{H}\right)_{j-1 / 2, \ell-1 / 2} & :=\frac{w_{j-1, \ell-1}+w_{j-1, \ell}+w_{j, \ell-1}+w_{j, \ell}}{4},
\end{aligned}
$$

for $\left(x_{j-1 / 2}, y_{\ell-1 / 2}\right) \in \Omega_{H}$. These last three quantities are zero on $\partial \Omega_{H} . R_{H}$ and $R_{G_{H}}$ are the operators that define pointwise restrictions to $\bar{\Omega}_{H}$ and $G_{H} \cap \Omega$, respectively.

In the sequel we need norms for grid functions. To this end we introduce in the next section discrete versions of the Sobolev spaces $L^{2}(\Omega), W_{0}^{1,2}(\Omega)$ and $W^{2,2}(\Omega) \cap W_{0}^{1,2}(\Omega)$.

\section{Discrete $W^{m, 2}(\Omega)$ spaces}

For grid functions defined on $S_{H}$ we define

$$
\begin{aligned}
\left|w_{H}\right|_{0, H}^{2}:= & \sum_{j=1}^{N} \sum_{\ell=1}^{M} h_{j-1} k_{\ell-1}\left|w_{j-1 / 2, \ell-1 / 2}\right|^{2}, \\
\left|w_{H}\right|_{1, H}^{2}:= & \sum_{j=0}^{N} \sum_{\ell=1}^{M} h_{j-1 / 2} k_{\ell-1}\left|\left(\delta_{x} w_{H}\right)_{j, \ell-1 / 2}\right|^{2}+\sum_{j=1}^{N} \sum_{\ell=0}^{M} h_{j-1} k_{\ell-1 / 2}\left|\left(\delta_{y} w_{H}\right)_{j-1 / 2, \ell}\right|^{2}, \\
\left|w_{H}\right|_{2, H}^{2}:= & \sum_{j=1}^{N} \sum_{\ell=1}^{M} h_{j-1} k_{\ell-1}\left(\left|\left(\delta_{x}^{2} w_{H}\right)_{j-1 / 2, \ell-1 / 2}\right|^{2}+\left|\left(\delta_{y}^{2} w_{H}\right)_{j-1 / 2, \ell-1 / 2}\right|^{2}\right) \\
& +2 \sum_{j=0}^{N} \sum_{\ell=0}^{M} h_{j-1 / 2} k_{\ell-1 / 2}\left|\left(\delta_{x y} w_{H}\right)_{j, \ell}\right|^{2},
\end{aligned}
$$

with $\delta_{x y}$ given by $\left(\delta_{x y} w_{H}\right)_{j, \ell}:=\left(\left(\delta_{x} w_{H}\right)_{j, \ell+1 / 2}-\left(\delta_{x} w_{H}\right)_{j, \ell-1 / 2}\right) / k_{\ell-1 / 2}$. Let us now introduce discrete counterparts of $L^{2}(\Omega), W_{0}^{1,2}(\Omega)$ and $W^{2,2}(\Omega) \cap W_{0}^{1,2}(\Omega)$.

We are going to consider the extension on $S_{H} \backslash \bar{\Omega}_{H}$ by zero for grid functions defined on $\bar{\Omega}_{H}$. When it is clear from the context that we use the extended function we use the same notation as for the function on $\bar{\Omega}_{H}$.

We denote by $\stackrel{\circ}{L}_{H}^{2}(\Omega), \stackrel{\circ}{W}_{H}^{1,2}(\Omega)$ and $W_{H}^{2,2}(\Omega) \cap \stackrel{\circ}{W}_{H}^{1,2}(\Omega)$, respectively, the space of functions defined on $\bar{\Omega}_{H}$ which are zero on $\partial \Omega_{H}$ equipped with the norm $\|\cdot\|_{0, H},\|\cdot\|_{1, H}$ and $\|\cdot\|_{2, H}$,

$$
\left\|v_{H}\right\|_{m, H}:=\left(\sum_{r=0}^{m}\left|v_{H}\right|_{r, H}^{2}\right)^{1 / 2}, \quad m=0,1,2 .
$$


The symbol $\|\cdot\|_{0, H}$ does not always represent a norm in spaces of grid functions. In order to overcome this fact we restrict the definition to spaces of grid functions which are zero in $\partial \Omega_{H}$. The space $\stackrel{\circ}{L}_{H}^{2}(\Omega)$ is endowed with the inner product

$$
\left(v_{H}, w_{H}\right)_{H}:=\sum_{j=1}^{N} \sum_{\ell=1}^{M} h_{j-1} k_{\ell-1}\left(v_{H}\right)_{j-1 / 2, \ell-1 / 2}\left(\bar{w}_{H}\right)_{j-1 / 2, \ell-1 / 2},
$$

which is a discrete version of the usual $L^{2}(\Omega)$-inner product, $(\cdot, \cdot)_{0}$. The spaces $\stackrel{\circ}{L}_{H}^{2}(\Omega)$ and $\stackrel{\circ}{W}_{H}^{1,2}(\Omega)$ form discrete approximations of $L^{2}(\Omega)$ and $W_{0}^{1,2}(\Omega)$, respectively, in the sense explained in what follows [29,30].

Let $\Lambda$ be a sequence of positive vectors of step-sizes, $H=(h, k)$, such that the maximum step-size, $H_{\max }$, converges to zero. A sequence $\left(v_{H}\right)_{\Lambda} \in \Pi \stackrel{\circ}{L}_{H}^{2}(\Omega)$ converges discretely to $v, v_{H} \rightarrow v$, in $\left(L^{2}(\Omega), \Pi \stackrel{\circ}{L}_{H}^{2}(\Omega)\right)(H \in \Lambda)$, if for each $\epsilon>0$ there exists $\varphi \in C^{\infty}(\Omega)$ such that

$$
\|v-\varphi\|_{L^{2}(\Omega)} \leqslant \epsilon, \quad \lim _{H_{\max } \rightarrow 0} \sup \left\{\left\|v_{H}-R_{H} \varphi\right\|_{0, H}\right\} \leqslant \epsilon .
$$

A sequence $\left(v_{H}\right)_{\Lambda} \in \Pi \stackrel{\circ}{W}_{H}^{1,2}(\Omega)$ converges discretely to $v \in W_{0}^{1,2}(\Omega), v_{H} \rightarrow v$, in $\left(W_{0}^{1,2}(\Omega), \Pi \stackrel{\circ}{W}_{H}^{1,2}(\Omega)\right)(H \in \Lambda)$, if for each $\epsilon>0$ there exists $\varphi \in C^{\infty}(\Omega)$ such that

$$
\|v-\varphi\|_{W^{1,2}(\Omega)} \leqslant \epsilon, \quad \lim _{H_{\max } \rightarrow 0} \sup \left\{\left\|v_{H}-R_{H} \varphi\right\|_{1, H}\right\} \leqslant \epsilon .
$$

A sequence $\left(v_{H}\right)_{\Lambda}$ converges weakly to $v, v_{H} \rightarrow v$, in $\left(L^{2}(\Omega), \Pi \stackrel{\circ}{L}_{H}^{2}(\Omega)\right)(H \in \Lambda)$, if

$$
\left(w_{H}, v_{H}\right)_{H} \rightarrow(w, v)_{0} \quad(H \in \Lambda)
$$

for all $w \in L^{2}(\Omega)$ and $\left(w_{H}\right)_{\Lambda} \in \Pi \stackrel{\circ}{L}_{H}^{2}(\Omega)$ such that $\left(w_{H}\right)_{\Lambda}$ converges weakly to $w$ in $\left(L^{2}(\Omega), \Pi \stackrel{\circ}{L}_{H}^{2}(\Omega)\right)$. The following lemma [1] is an important technical tool in the stability analysis which uses the concept of discrete compactness [30].

Lemma 1. Let $\left(v_{H}\right)_{\Lambda} \in \Pi \stackrel{\circ}{W}_{H}^{1,2}(\Omega)$ be a bounded sequence. Then there exists a subsequence $\Lambda^{\prime} \subset \Lambda$ such that $\left(v_{H}\right)_{\Lambda^{\prime}} \in \Pi \stackrel{\circ}{L}_{H}^{2}(\Omega)$ converges discretely in $\left(L^{2}(\Omega), \Pi \stackrel{\circ}{L}_{H}^{2}(\Omega)\right)\left(H \in \Lambda^{\prime}\right)$. Moreover, if

$$
v_{H} \rightarrow v \quad \text { in }\left(L^{2}(\Omega), \Pi \stackrel{\circ}{L}_{H}^{2}(\Omega)\right) \quad(H \in \Lambda)
$$

then $v \in W_{0}^{1,2}(\Omega)$.

The proof in [1] is specific for the normed spaces of cell-centered grid functions which we consider in this paper. For general results of discrete compactness in spaces of vertex-centered grid functions we cite [14]. The proof of Lemma 2 can be found in [29].

Lemma 2. Let $\left(v_{H}\right)_{\Lambda} \in \Pi \stackrel{\circ}{L}_{H}^{2}(\Omega)$ be a bounded sequence. Then there exists a subsequence $\Lambda^{\prime} \subset \Lambda$ and an element $v \in L^{2}(\Omega)$ such that

$$
v_{H} \rightarrow v \quad \text { in }\left(L^{2}(\Omega), \Pi \stackrel{\circ}{L}_{H}^{2}(\Omega)\right) \quad\left(H \in \Lambda^{\prime}\right) .
$$

\section{Stability}

Our aim in this section is to show an inverse stability condition for $A_{H}$.

We first define the operator $A_{H}^{*}: W_{H}^{2,2}(\Omega) \cap \stackrel{\circ}{W_{H}^{1,2}}(\Omega) \rightarrow \stackrel{\circ}{L}{ }_{H}^{2}(\Omega), A_{H}^{*}:=A_{H}^{(2) *}+A_{H}^{(1) *}$, with $A_{H}^{(1) *} v_{H}:=$ $A_{H}^{(2) *} v_{H}:=0$ on $\partial \Omega_{H}$,

$$
\begin{aligned}
& A_{H}^{(2) *} v_{H}:=-\delta_{x}\left(a \delta_{x} v_{H}\right)-\delta_{y}\left(c \delta_{y} v_{H}\right) \quad \text { in } \Omega_{H}, \\
& A_{H}^{(1) *} v_{H}:=-\delta_{x}\left(\bar{d} M_{x}^{*} v_{H}\right)-\delta_{y}\left(\bar{e} M_{y}^{*} v_{H}\right)+\bar{f} v_{H} \quad \text { in } \Omega_{H},
\end{aligned}
$$


where

$$
\begin{aligned}
\left(M_{x}^{*} v_{H}\right)_{j, \ell-1 / 2} & :=\frac{v_{j-1 / 2, \ell-1 / 2} h_{j-1}+v_{j+1 / 2, \ell-1 / 2} h_{j}}{2 h_{j-1 / 2}}, \\
\left(M_{y}^{*} v_{H}\right)_{j-1 / 2, \ell} & :=\frac{v_{j-1 / 2, \ell-1 / 2} k_{\ell-1}+v_{j-1 / 2, \ell+1 / 2} k_{\ell}}{2 k_{\ell-1 / 2}} .
\end{aligned}
$$

Before we prove the desired stability inequality we present the proof of the following result: there exists $C>0$ such that

$$
\left\|v_{H}\right\|_{1, H} \leqslant C\left\|A_{H}^{*} v_{H}\right\|_{0, H} \quad \forall v_{H} \in W_{H}^{2,2}(\Omega) \cap \stackrel{\circ}{W}_{H}^{1,2}(\Omega) .
$$

This will be made with the aid of Lemmas 3-6. The first result is obvious using the definitions.

Lemma 3. If $\left(v_{H}\right)_{\Lambda} \in \Pi \stackrel{\circ}{W}{ }_{H}^{1,2}(\Omega)$ is a bounded sequence and $\alpha \in C(\bar{\Omega})$ then $\left(M_{x}\left(\alpha \delta_{x} v_{H}\right)\right)_{\Lambda}$ and $\left(M_{y}\left(\alpha \delta_{y} v_{H}\right)\right)_{\Lambda}$ are bounded on $\Pi \stackrel{\circ}{L}_{H}^{2}(\Omega)$.

Lemma 4. Let $H \in \Lambda$. Then there exists positive constants $C_{1}, C_{2}$ and $C_{3}$ not depending on $H$ such that

$$
\left(-\delta_{x}\left(a \delta_{x} v_{H}\right)-\delta_{y}\left(c \delta_{y} v_{H}\right), v_{H}\right)_{H} \geqslant C_{1}\left\|v_{H}\right\|_{1, H}^{2} \quad \forall v_{H} \in \stackrel{\circ}{W}_{H}^{1,2}(\Omega),
$$

and

$$
\left(A_{H} v_{H}, v_{H}\right)_{H} \geqslant C_{2}\left\|v_{H}\right\|_{1, H}^{2}-C_{3}\left\|v_{H}\right\|_{0, H}^{2} \quad \forall v_{H} \in \stackrel{\circ}{W}_{H}^{1,2}(\Omega) .
$$

Proof. Since $a$ has a lower bound $\underline{a}$,

$$
\begin{aligned}
\underline{a} & \sum_{j=0}^{N} \sum_{\ell=1}^{M} h_{j-1 / 2} k_{\ell-1}\left|\left(\delta_{x} v_{H}\right)_{j, \ell-1 / 2}\right|^{2} \\
\leqslant & \sum_{j=0}^{N} \sum_{\ell=1}^{M} h_{j-1 / 2} k_{\ell-1} a\left(x_{j}, y_{\ell-1 / 2}\right)\left|\left(\delta_{x} v_{H}\right)_{j, \ell-1 / 2}\right|^{2} \\
= & \sum_{j=1}^{N-1} \sum_{\ell=1}^{M} k_{\ell-1} a\left(x_{j}, y_{\ell-1 / 2}\right)\left(\delta_{x} v_{H}\right)_{j, \ell-1 / 2}\left(\bar{v}_{j+1 / 2, \ell-1 / 2}-\bar{v}_{j-1 / 2, \ell-1 / 2}\right) \\
& +\sum_{\ell=1}^{M} k_{\ell-1} a\left(x_{0}, y_{\ell-1 / 2}\right)\left(\delta_{x} v_{H}\right)_{0, \ell-1 / 2} \bar{v}_{1 / 2, \ell-1 / 2}-\sum_{\ell=1}^{M} k_{\ell-1} a\left(x_{N}, y_{\ell-1 / 2}\right)\left(\delta_{x} v_{H}\right)_{N, \ell-1 / 2} \bar{v}_{N-1 / 2, \ell-1 / 2,},
\end{aligned}
$$

and then

$$
\begin{aligned}
\left(-\delta_{x}\left(a \delta_{x} v_{H}\right), v_{H}\right)_{H} & =-\sum_{j=1}^{N} \sum_{\ell=1}^{M} k_{\ell-1}\left(\left(a \delta_{x} v_{H}\right)_{j, \ell-1 / 2}-\left(a \delta_{x} v_{H}\right)_{j-1, \ell-1 / 2}\right) \bar{v}_{j-1 / 2, \ell-1 / 2} \\
& \geqslant \underline{a} \sum_{j=0}^{N} \sum_{\ell=1}^{M} h_{j-1 / 2} k_{\ell-1}\left|\left(\delta_{x} v_{H}\right)_{j, \ell-1 / 2}\right|^{2} .
\end{aligned}
$$

In the same way we can prove a correspondent estimate for $\left(-\delta_{y}\left(c \delta_{y} v_{H}\right), v_{H}\right)_{H}$. Then (7) follows. From Lemma 3 and using a discrete version of the Poincaré-Friedrichs inequality which is simple to prove, there exists $C_{4}>0$ such that

$$
\left|\left(M_{x}\left(d \delta_{x} v_{H}\right)+M_{y}\left(e \delta_{y} v_{H}\right)+f v_{H}, v_{H}\right)_{H}\right| \leqslant C_{4}\left\|v_{H}\right\|_{1, H}\left\|v_{H}\right\|_{0, H} .
$$

We conclude (8) using the fact that there exists $C_{3}>0$ such that

$$
C_{4}\left\|v_{H}\right\|_{1, H}\left\|v_{H}\right\|_{0, H} \leqslant \frac{C_{1}}{2}\left\|v_{H}\right\|_{1, H}^{2}+C_{3}\left\|v_{H}\right\|_{0, H}^{2} .
$$


Lemma 5. Let $\left(v_{H}\right)_{\Lambda} \in \Pi \stackrel{\circ}{W}_{H}^{1,2}(\Omega)$ and $v \in W_{0}^{1,2}(\Omega)$ such that

$$
v_{H} \rightarrow v \quad \text { in }\left(W_{0}^{1,2}(\Omega), \Pi \stackrel{\circ}{W}_{H}^{1,2}(\Omega)\right) \quad(H \in \Lambda)
$$

and let $\alpha \in C(\bar{\Omega})$. Then

$$
M_{x}\left(\alpha \delta_{x} v_{H}\right) \rightarrow \alpha v_{x} \quad \text { and } \quad M_{y}\left(\alpha \delta_{y} v_{H}\right) \rightarrow \alpha v_{y} \quad \text { in }\left(L^{2}(\Omega), \Pi \stackrel{\circ}{L}_{H}^{2}(\Omega)\right)(H \in \Lambda) .
$$

Proof. Let $C$ satisfy $\|\alpha\|_{L^{\infty}(\Omega)} \leqslant C$. For any positive real number $\epsilon$ there exists $\varphi \in C^{\infty}(\bar{\Omega})$ such that

$$
\|v-\varphi\|_{W^{1,2}(\Omega)} \leqslant \epsilon, \quad \lim _{H_{\max } \rightarrow 0} \sup \left\{\left\|v_{H}-R_{H} \varphi\right\|_{1, H}\right\} \leqslant \frac{1}{4 C} \epsilon .
$$

Since

$$
\left\|M_{x}\left(\alpha \delta_{x} v_{H}\right)-M_{x}\left(\alpha \delta_{x} R_{H} \varphi\right)\right\|_{0, H} \leqslant 2\|\alpha\|_{L^{\infty}(\Omega)}\left\|v_{H}-R_{H} \varphi\right\|_{1, H}
$$

and for $H_{\max }$ small enough

$$
\left\|M_{x}\left(\alpha \delta_{x} R_{H} \varphi\right)-R_{H}\left(\alpha \varphi_{x}\right)\right\|_{0, H} \leqslant \frac{\epsilon}{2},
$$

there exists a final section $\Lambda^{\prime} \subset \Lambda$ such that, for $H \in \Lambda^{\prime}$,

$$
\left\|M_{x}\left(\alpha \delta_{x} v_{H}\right)-R_{H}\left(\alpha \varphi_{x}\right)\right\|_{0, H} \leqslant \epsilon .
$$

Lemma 6. Let $\left(v_{H}\right)_{\Lambda}$ be a bounded sequence in $\Pi \stackrel{\circ}{W}_{H}^{1,2}(\Omega)$ and $\alpha \in C(\bar{\Omega})$. Then there exists a subsequence $\Lambda^{\prime} \subseteq \Lambda$ and an element $v \in W_{0}^{1,2}(\Omega)$ such that

$$
v_{H} \rightarrow v \quad \text { in }\left(L^{2}(\Omega), \Pi \stackrel{\circ}{L}_{H}^{2}(\Omega)\right)\left(H \in \Lambda^{\prime}\right)
$$

and the following weak convergence hold

$$
M_{x}\left(\alpha \delta_{x} v_{H}\right) \rightarrow \alpha v_{x} \quad \text { and } \quad M_{y}\left(\alpha \delta_{y} v_{H}\right) \rightarrow \alpha v_{y} \quad \text { in }\left(L^{2}(\Omega), \Pi \stackrel{\circ}{L}_{H}^{2}(\Omega)\right)\left(H \in \Lambda^{\prime}\right) .
$$

Proof. It follows from Lemma 3 that $\left(M_{x}\left(\alpha \delta_{x} v_{H}\right)\right)_{\Lambda}$ is bounded on $\stackrel{\circ}{L}_{H}^{2}(\Omega)$. Taking Lemma 2 into account we have

$$
\left(M_{x}\left(\alpha \delta_{x} v_{H}\right)\right)_{\Lambda} \rightarrow w \quad \text { in }\left(L^{2}(\Omega), \Pi \stackrel{\circ}{L}_{H}^{2}(\Omega)\right)\left(H \in \Lambda^{\prime \prime}\right),
$$

for a subsequence $\Lambda^{\prime \prime} \subseteq \Lambda$ and $w \in L^{2}(\Omega)$. Then for any $\varphi \in C_{0}^{\infty}(\Omega)$

$$
\left(R_{H} \varphi, M_{x}\left(\alpha \delta_{x} v_{H}\right)\right)_{H} \rightarrow(\varphi, w)_{0} \quad\left(H \in \Lambda^{\prime \prime}\right) .
$$

From Lemma 1, there exists $v \in W_{0}^{1,2}(\Omega)$ and $\Lambda^{\prime} \subseteq \Lambda^{\prime \prime}$, such that

$$
v_{H} \rightarrow v \quad \text { in }\left(L^{2}(\Omega), \Pi \stackrel{\circ}{L}_{H}^{2}(\Omega)\right)\left(H \in \Lambda^{\prime}\right) .
$$

Let us prove that

$$
\delta_{x}\left(\alpha M_{x}^{*} R_{H} \varphi\right) \rightarrow(\alpha \varphi)_{x} \quad \text { in }\left(L^{2}(\Omega), \Pi \stackrel{\circ}{L}_{H}^{2}(\Omega)\right) \quad\left(H \in \Lambda^{\prime}\right),
$$

with $\left(M_{x}^{*} R_{H} \varphi\right)_{j, \ell-1 / 2}$ given by (6). Let $\psi \in C_{0}^{\infty}(\Omega)$. From Lemma 5

$$
\begin{aligned}
\left(-\delta_{x}\left(\alpha M_{x}^{*} R_{H} \varphi\right), R_{H} \psi\right)_{H} & =\left(R_{H} \varphi, M_{x}\left(\alpha \delta_{x} R_{H} \psi\right)\right)_{H} \\
& \rightarrow\left(\varphi, \alpha \psi_{x}\right)_{0},
\end{aligned}
$$

or equivalently,

$$
\left(-\delta_{x}\left(\alpha M_{x}^{*} R_{H} \varphi\right), R_{H} \psi\right)_{H} \rightarrow\left(-(\alpha \varphi)_{x}, \psi\right)_{0} .
$$

From Lemma 2, there exists $z \in L^{2}(\Omega)$ such that 


$$
\delta_{x}\left(\alpha M_{x}^{*} R_{H} \varphi\right) \rightarrow z \quad \text { in }\left(L^{2}(\Omega), \Pi^{\circ}{ }_{H}^{2}(\Omega)\right)\left(H \in \Lambda^{\prime}\right)
$$

and consequently

$$
\left(-\delta_{x}\left(\alpha M_{x}^{*} R_{H} \varphi\right), R_{H} \psi\right)_{H} \rightarrow(-z, \psi)_{0} .
$$

From (11) and (12) we obtain (10). Since

$$
\begin{aligned}
\left(R_{H} \varphi, M_{x}\left(\alpha \delta_{x} v_{H}\right)\right)_{H} & =\left(-\delta_{x}\left(\alpha M_{x}^{*} R_{H} \varphi\right), v_{H}\right)_{H} \\
& \rightarrow\left(-(\alpha \varphi)_{x}, v\right)_{0}=\left(\varphi, \alpha v_{x}\right)_{0},
\end{aligned}
$$

using (9) we conclude that

$$
M_{x}\left(\alpha \delta_{x} v_{H}\right) \rightarrow \alpha v_{x} \quad \text { in }\left(L^{2}(\Omega), \Pi \stackrel{\circ}{L}_{H}^{2}(\Omega)\right)\left(H \in \Lambda^{\prime}\right) .
$$

Theorem 1. There exists a final sequence $\Lambda^{\prime} \subset \Lambda$ and a constant $C>0$ not depending on $H$ such that

$$
\left\|v_{H}\right\|_{1, H} \leqslant C\left\|A_{H}^{*} v_{H}\right\|_{0, H} \quad \forall v_{H} \in W_{H}^{2,2}(\Omega) \cap \stackrel{\circ}{W}_{H}^{1,2}(\Omega), H \in \Lambda^{\prime} .
$$

Proof. Assuming (13) not to hold we can find a subsequence $\Lambda^{\prime \prime} \subseteq \Lambda$ and elements $v_{H}, H \in \Lambda^{\prime \prime}$, such that

$\left\|v_{H}\right\|_{1, H}=1 \quad$ and $\quad\left\|A_{H}^{*} v_{H}\right\|_{0, H} \rightarrow 0 \quad\left(H \in \Lambda^{\prime \prime}\right)$.

Lemma 1 allow the sequence $\Lambda^{\prime \prime}$ and $v \in W_{0}^{1,2}(\Omega)$ to be chosen such that $\left(v_{H}\right)_{\Lambda^{\prime \prime}}$ converges discretely to $v$ in $\left(L^{2}(\Omega), \Pi \stackrel{\circ}{L}_{H}^{2}(\Omega)\right)$. Let $w \in W_{0}^{1,2}(\Omega)$ be the solution of

$$
\left(a w_{x}, z_{x}\right)_{0}+\left(c w_{y}, z_{y}\right)_{0}=\left((d v)_{x}+(e v)_{y}+f v, z\right)_{0} \quad \forall z \in W_{0}^{1,2}(\Omega)
$$

and $\left(w_{H}\right)_{\Lambda} \in \Pi \stackrel{\circ}{W}_{H}^{1,2}(\Omega)$ such that $w_{H} \rightarrow w$ in $\left(W_{0}^{1,2}(\Omega), \Pi \stackrel{\circ}{W}_{H}^{1,2}(\Omega)\right)(H \in \Lambda)$. Let us prove the convergence

$$
\left|z_{H}\right|_{1, H} \rightarrow 0,
$$

for $z_{H}=v_{H}-w_{H}$. Lemma 4 gives the existence of $C>0$ such that

$$
\left|z_{H}\right|_{1, H}^{2} \leqslant C\left(\left(A_{H}^{*} v_{H}, z_{H}\right)_{H}+a\left(w_{H}, z_{H}\right)+c\left(w_{H}, z_{H}\right)+\left(v_{H}, A_{H}^{(1)} z_{H}\right)_{H}\right)
$$

where

$$
\begin{aligned}
& a\left(w_{H}, z_{H}\right):=\sum_{j=0}^{N} \sum_{\ell=1}^{M} h_{j-1 / 2} k_{\ell-1} a\left(x_{j}, y_{\ell-1 / 2}\right)\left(\delta_{x} w_{H}\right)_{j, \ell-1 / 2}\left(\delta_{x} \bar{z}_{H}\right)_{j, \ell-1 / 2}, \\
& c\left(w_{H}, z_{H}\right):=\sum_{j=1}^{N} \sum_{\ell=0}^{M} h_{j-1} k_{\ell-1 / 2} c\left(x_{j-1 / 2}, y_{\ell}\right)\left(\delta_{y} w_{H}\right)_{j-1 / 2, \ell}\left(\delta_{y} \bar{z}_{H}\right)_{j-1 / 2, \ell}, \\
& A_{H}^{(1)} z_{H}:=M_{x}\left(d \delta_{x} z_{H}\right)+M_{y}\left(e \delta_{y} z_{H}\right)+f z_{H} .
\end{aligned}
$$

Since $\left\|A_{H}^{*} v_{H}\right\|_{0, H} \rightarrow 0$, it follows that $\left(A_{H}^{*} v_{H}, z_{H}\right)_{H} \rightarrow 0$. Let $z=v-w$. Our aim is to prove that

$$
a\left(w_{H}, z_{H}\right) \rightarrow\left(a w_{x}, z_{x}\right)_{0}, \quad c\left(w_{H}, z_{H}\right) \rightarrow\left(c w_{y}, z_{y}\right)_{0} \quad\left(H \in \Lambda^{\prime \prime}\right) .
$$

Lemma 5 yields

$$
M_{x}\left(\delta_{x} w_{H}\right) \rightarrow w_{x} \quad \text { in }\left(L^{2}(\Omega), \Pi \stackrel{\circ}{L}_{H}^{2}(\Omega)\right)(H \in \Lambda) .
$$

Since $\left(z_{H}\right)_{\Lambda}, H \in \Lambda^{\prime \prime}$, is bounded on $\Pi \stackrel{\circ}{W}_{H}^{1,2}(\Omega)$, Lemma 6 allows a subsequence $\Lambda^{\prime} \subset \Lambda^{\prime \prime}$ to be chosen such that

$$
\left(M_{x}\left(a \delta_{x} z_{H}\right)\right)_{\Lambda} \rightarrow a z_{x}, \quad\left(M_{y}\left(c \delta_{y} z_{H}\right)\right)_{\Lambda} \rightarrow c z_{y} \quad \text { in }\left(L^{2}(\Omega), \Pi \stackrel{\circ}{L}_{H}^{2}(\Omega)\right)\left(H \in \Lambda^{\prime}\right),
$$

and consequently (18) holds. For the last term of (17) we have

$$
\left(v_{H}, A_{H}^{(1)} z_{H}\right)_{H} \rightarrow\left(v, A^{(1)} z\right)_{0} \quad\left(H \in \Lambda^{\prime \prime}\right) .
$$


Since $w$ is the solution of (15), it follows that

$$
a\left(w_{H}, z_{H}\right)+c\left(w_{H}, z_{H}\right)+\left(v_{H}, A_{H}^{(1)} z_{H}\right)_{H} \rightarrow 0 \quad\left(H \in \Lambda^{\prime \prime}\right)
$$

and (16) follows. Then

$$
v_{H}=z_{H}+w_{H} \rightarrow w \quad \text { in }\left(L^{2}(\Omega), \Pi \stackrel{\circ}{L}_{H}^{2}(\Omega)\right)\left(H \in \Lambda^{\prime}\right),
$$

and

$$
(A w, z)_{0}=0 \quad \forall z \in W_{0}^{1,2}(\Omega) .
$$

For $A$ being injective $\left\|v_{H}\right\|_{1, H}=1$ is not possible.

Let us now prove a stability result for $A_{H}^{(2) *}$.

Lemma 7. There exists $C$ not depending on $H$ such that, for $H \in \Lambda$,

$$
\left\|v_{H}\right\|_{2, H} \leqslant C\left(\left\|A_{H}^{(2) *} v_{H}\right\|_{0, H}+\left\|v_{H}\right\|_{1, H}\right) \quad \forall v_{H} \in W_{H}^{2,2}(\Omega) \cap \stackrel{\circ}{W}_{H}^{1,2}(\Omega) .
$$

Proof. Let $v_{H} \in W_{H}^{2,2}(\Omega) \cap \stackrel{\circ}{W}{ }_{H}^{1,2}(\Omega)$. We define

$$
\begin{aligned}
B_{x}^{(1)} v_{H}:= & \sum_{j=1}^{N} \sum_{\ell=1}^{M} h_{j-1} k_{\ell-1}\left(\delta_{x}^{2} \bar{v}_{H}\right)_{j-1 / 2, \ell-1 / 2}\left[\frac{a\left(x_{j-1 / 2}, y_{\ell-1 / 2}\right)-a\left(x_{j-1}, y_{\ell-1 / 2}\right)}{h_{j-1}}\left(\delta_{x} v_{H}\right)_{j-1, \ell-1 / 2}\right. \\
& \left.+\frac{a\left(x_{j}, y_{\ell-1 / 2}\right)-a\left(x_{j-1 / 2}, y_{\ell-1 / 2}\right)}{h_{j-1}}\left(\delta_{x} v_{H}\right)_{j, \ell-1 / 2}\right] \\
& +\sum_{j=0}^{N} \sum_{\ell=0}^{M} h_{j-1} k_{\ell-1 / 2} \frac{c\left(x_{j}, y_{\ell}\right)-c\left(x_{j-1 / 2}, y_{\ell}\right)}{h_{j-1}}\left(\delta_{y} v_{H}\right)_{j-1 / 2, \ell}\left(\delta_{x y} \bar{v}_{H}\right)_{j, \ell} \\
& +\sum_{j=0}^{N} \sum_{\ell=0}^{M} h_{j} k_{\ell-1 / 2} \frac{c\left(x_{j+1 / 2}, y_{\ell}\right)-c\left(x_{j}, y_{\ell}\right)}{h_{j}}\left(\delta_{y} v_{H}\right)_{j+1 / 2, \ell}\left(\delta_{x y} \bar{v}_{H}\right)_{j, \ell}, \\
B_{x}^{(2)} v_{H}:= & \sum_{j=1}^{N} \sum_{\ell=1}^{M} h_{j-1} k_{\ell-1} a\left(x_{j-1 / 2}, y_{\ell-1 / 2}\right)\left|\left(\delta_{x}^{2} v_{H}\right)_{j-1 / 2, \ell-1 / 2}\right|^{2} \\
& +\sum_{j=0}^{N} \sum_{\ell=0}^{M} h_{j-1 / 2} k_{\ell-1 / 2} c\left(x_{j}, y_{\ell}\right)\left|\left(\delta_{x y} v_{H}\right)_{j, \ell}\right|^{2},
\end{aligned}
$$

$B_{y}^{(1)}$ and $B_{y}^{(2)}$ similar to $B_{x}^{(1)}$ and $B_{x}^{(2)}$, respectively, replacing $a$ with $c, x$ with $y$ and the indexes in a obvious way. We have

$$
\left(A_{H}^{(2) *} v_{H}, \delta_{x}^{2} v_{H}+\delta_{y}^{2} v_{H}\right)_{H}=-B_{H}^{(1)} v_{H}-B_{H}^{(2)} v_{H},
$$

where $B_{H}^{(1)}:=B_{x}^{(1)}+B_{y}^{(1)}$ and $B_{H}^{(2)}:=B_{x}^{(2)}+B_{y}^{(2)}$. The conditions assumed for $a$ and $c$ give the existence of $C_{E}>0$ and $C_{L}>0$ such that

$$
C_{E}\left|v_{H}\right|_{2, H}^{2} \leqslant B_{H}^{(2)} v_{H} \quad \text { and } \quad B_{H}^{(1)} v_{H} \leqslant C_{L}\left|v_{H}\right|_{1, H}\left|v_{H}\right|_{2, H},
$$

which together with (20) yield

$$
\begin{aligned}
C_{E}\left|v_{H}\right|_{2, H}^{2} & \leqslant\left|\left(A_{H}^{(2) *} v_{H}, \delta_{x}^{2} v_{H}+\delta_{y}^{2} v_{H}\right)_{H}\right|+\left|B_{H}^{(1)} v_{H}\right| \\
& \leqslant\left\|A_{H}^{(2) *} v_{H}\right\|_{0, H}\left|v_{H}\right|_{2, H}+C_{L}\left|v_{H}\right|_{1, H}\left|v_{H}\right|_{2, H} .
\end{aligned}
$$

Then (19) follows with $C=\max \left\{1 / C_{E}, C_{L} / C_{E}\right\}$.

The main result of this section is the following stability theorem. 
Theorem 2. There exists $C>0$ and a final section $\Lambda^{\prime} \subset \Lambda$ such that

$$
\left\|v_{H}\right\|_{0, H} \leqslant C \sup _{0 \neq w_{H} \in W_{H}^{2,2}(\Omega) \cap \stackrel{\circ}{H}_{H}^{1,2}(\Omega)} \frac{\left|\left(A_{H} v_{H}, w_{H}\right)_{H}\right|}{\left\|w_{H}\right\|_{2, H}}
$$

$\forall v_{H} \in W_{H}^{2,2}(\Omega) \cap \stackrel{\circ}{W}_{H}^{1,2}(\Omega), H \in \Lambda^{\prime}$.

Proof. Let $v_{H} \in W_{H}^{2,2}(\Omega) \cap \stackrel{\circ}{W} \underset{H}{1,2}(\Omega)$. Since $A_{H}^{(1) *}: \stackrel{\circ}{W}_{H}^{1,2}(\Omega) \rightarrow \stackrel{\circ}{L}_{H}^{2}(\Omega)$ is bounded, there exists $C_{L}>0$ such that

$$
\left\|A_{H}^{(2) *} v_{H}\right\|_{0, H} \leqslant\left\|A_{H}^{*} v_{H}\right\|_{0, H}+\left\|A_{H}^{(1) *} v_{H}\right\|_{0, H} \leqslant\left\|A_{H}^{*} v_{H}\right\|_{0, H}+C_{L}\left\|v_{H}\right\|_{1, H} .
$$

Lemma 7 gives the existence of $C^{\prime}>0$ such that

$$
\begin{aligned}
\left\|v_{H}\right\|_{2, H} & \leqslant C^{\prime}\left(\left\|A_{H}^{(2) *} v_{H}\right\|_{0, H}+\left\|v_{H}\right\|_{1, H}\right) \\
& \leqslant C^{\prime}\left\|A_{H}^{*} v_{H}\right\|_{0, H}+\left(C^{\prime}+C^{\prime} C_{L}\right)\left\|v_{H}\right\|_{1, H} .
\end{aligned}
$$

The existence of $C>0$ such that

$$
\left\|v_{H}\right\|_{2, H} \leqslant C\left\|A_{H}^{*} v_{H}\right\|_{0, H} \quad \forall v_{H} \in W_{H}^{2,2}(\Omega) \cap \stackrel{\circ}{W}{ }_{H}^{1,2}(\Omega)
$$

follows from Theorem 1 . Then $\left(A_{H}^{*}\right)^{-1}: \stackrel{\circ}{L}_{H}^{2}(\Omega) \rightarrow W_{H}^{2,2}(\Omega) \cap \stackrel{\circ}{W}_{H}^{1,2}(\Omega)$ exists and (22) is equivalent to

$$
\left\|\left(A_{H}^{*}\right)^{-1} \varphi_{H}\right\|_{2, H} \leqslant C\left\|\varphi_{H}\right\|_{0, H} \quad \forall \varphi_{H} \in \stackrel{\circ}{L}_{H}^{2}(\Omega) .
$$

Combining this last inequality with

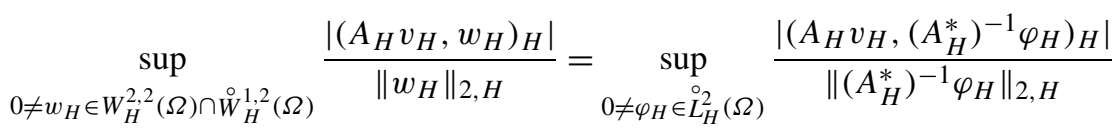

$$
\begin{aligned}
& =\sup _{0 \neq \varphi_{H} \in \stackrel{\circ}{L}_{H}^{2}(\Omega)} \frac{\left|\left(v_{H}, \varphi_{H}\right)_{H}\right|}{\left\|\left(A_{H}^{*}\right)^{-1} \varphi_{H}\right\|_{2, H}},
\end{aligned}
$$

results (21).

The estimate (21) can be given in an alternative form which uses a negative norm. We introduce the discrete Laplace operator

$$
\Delta_{H} v_{H}:=\delta_{x}^{2} v_{H}+\delta_{y}^{2} v_{H}, \quad v_{H} \in W_{H}^{2,2}(\Omega) \cap \stackrel{\circ}{W}_{H}^{1,2}(\Omega)
$$

and the norm

$$
\left\|v_{H}\right\|_{-\Delta_{H}}:=\sup _{0 \neq w_{H} \in W_{H}^{2,2}(\Omega) \cap \stackrel{\circ}{W}_{H}^{1,2}(\Omega)} \frac{\left|\left(v_{H}, w_{H}\right)_{H}\right|}{\left\|\Delta_{H} w_{H}\right\|_{0, H}},
$$

where the extension by zero on $S_{H} \backslash \bar{\Omega}_{H}$ of $v_{H}$ and $w_{H}$ is considered.

Some trivial algebraic manipulations lead to the next result [2]: the norms $\|\cdot\|_{2, H}$ and $\left\|\Delta_{H} \cdot\right\|_{0, H}$ are equivalent in $W_{H}^{2,2}(\Omega) \cap \stackrel{\circ}{W}_{H}^{1,2}(\Omega)$. With the definition (23) the estimate (21) is equivalent to

$$
\left\|v_{H}\right\|_{0, H} \leqslant C\left\|A_{H} v_{H}\right\|_{-\Delta_{H}} \quad \forall v_{H} \in W_{H}^{2,2}(\Omega) \cap \stackrel{\circ}{W}_{H}^{1,2}(\Omega) .
$$

\section{Convergence}

The main result of this paper in Theorem 3 relies on the stability result of Theorem 2. An estimate for $\left\|R_{H} u-u_{H}\right\|_{0, H}$ will be obtained with the aid of (21) replacing $v_{H}$ by $R_{H} u-u_{H}$ and bounding

$$
\left(A_{H}\left(R_{H} u\right)-M_{H}\left(R_{G_{H}} g\right), v_{H}\right)_{H} .
$$


The bounds in Lemmas 8-10 are for that purpose. The Bramble-Hilbert Lemma (see e.g. [5]) and Schwarz's inequality

$$
\|v\|_{L^{1}(\square)} \leqslant h^{1 / 2} k^{1 / 2}\|v\|_{L^{2}(\square)} \quad \forall v \in L^{2}(\square),
$$

being $h \times k$ the dimension of the rectangle $\square$, for estimating the local contributions measured in the $L^{1}$-norm in terms of $L^{2}$-norm, are the main technical tools to obtain the desired convergence order.

In what follows we use the notation $\sum_{\Omega_{H}}$ for the sum over the set of indexes $(j, \ell)$ such that $\left(x_{j+1 / 2}, y_{\ell+1 / 2}\right) \in \Omega_{H}$.

Lemma 8. Let $u \in H^{4}(\Omega)$. Then, for all $v_{H} \in W_{H}^{2,2}(\Omega) \cap \stackrel{\circ}{W}_{H}^{1,2}(\Omega)$ there holds

$$
\begin{aligned}
& \left|\left(-\delta_{x}\left(a \delta_{x} u\right), v_{H}\right)_{H}-\left(M_{H} R_{G_{H}}\left(a u_{x}\right)_{x}, v_{H}\right)_{H}\right| \\
& \quad \leqslant C\|a\|_{W^{3, \infty}(\Omega)}\left(\sum_{\Omega_{H}}\left(h_{j}^{2}+k_{\ell}^{2}\right)^{2}\|u\|_{H^{4}\left(\left(x_{j}, x_{j+1}\right) \times\left(y_{\ell}, y_{\ell+1}\right)\right)}^{2}\right)^{1 / 2}\left\|v_{H}\right\|_{2, H}, \\
& \left|\left(-\delta_{y}\left(c \delta_{y} u\right), v_{H}\right)_{H}-\left(M_{H} R_{G_{H}}\left(c u_{y}\right)_{y}, v_{H}\right)_{H}\right| \\
& \quad \leqslant C\|c\|_{W^{3, \infty}(\Omega)}\left(\sum_{\Omega_{H}}\left(h_{j}^{2}+k_{\ell}^{2}\right)^{2}\|u\|_{H^{4}\left(\left(x_{j}, x_{j+1}\right) \times\left(y_{\ell}, y_{\ell+1}\right)\right)}^{2}\right)^{1 / 2}\left\|v_{H}\right\|_{2, H} .
\end{aligned}
$$

Proof. Let $v_{H} \in W_{H}^{2,2}(\Omega) \cap \stackrel{\circ}{W}_{H}^{1,2}(\Omega)$. We consider, in first place, only the terms in

$$
\left(\delta_{x} a \delta_{x} u, v_{H}\right)_{H} \text { and }\left(M_{H} R_{G_{H}}\left(a u_{x}\right)_{x}, v_{H}\right)_{H}
$$

which have the factor $\bar{v}_{j+1 / 2, \ell+1 / 2}$, for some $j$, with $\ell$ given. Let us suppose, without loss of generality, that the set of the points in the form $\left(\cdot, y_{\ell+1 / 2}\right)$ belonging to $\Omega_{H}$ is

$$
\left\{\left(x_{p_{\ell}+1 / 2}, y_{\ell+1 / 2}\right),\left(x_{p_{\ell}+3 / 2}, y_{\ell+1 / 2}\right), \ldots,\left(x_{p_{\ell}+N_{\ell}-1 / 2}, y_{\ell+1 / 2}\right)\right\} .
$$

Let

$$
S_{1}:=\sum_{j=p_{\ell}}^{p_{\ell}+N_{\ell}-1} h_{j} k_{\ell}\left(\delta_{x} a \delta_{x} u\right)_{j+1 / 2, \ell+1 / 2} \bar{v}_{j+1 / 2, \ell+1 / 2}
$$

and

$$
S_{1}^{(1)}:=-\sum_{j=p_{\ell}}^{p_{\ell}+N_{\ell}}\left(\int_{y_{\ell}}^{y_{\ell+1}} \int_{x_{j-1 / 2}}^{x_{j+1 / 2}} a\left(x_{j}, y\right) u_{x}(x, y) d x d y\right)\left(\delta_{x} \bar{v}_{H}\right)_{j, \ell+1 / 2} .
$$

We have

$$
\begin{aligned}
S_{1} & =\sum_{j=p_{\ell}}^{p_{\ell}+N_{\ell}-1} k_{\ell}\left(\left(a \delta_{x} u\right)_{j+1, \ell+1 / 2}-\left(a \delta_{x} u\right)_{j, \ell+1 / 2}\right) \bar{v}_{j+1 / 2, \ell+1 / 2} \\
& =-\sum_{j=p_{\ell}}^{p_{\ell}+N_{\ell}} k_{\ell} \int_{x_{j-1 / 2}}^{x_{j+1 / 2}} a\left(x_{j}, y_{\ell+1 / 2}\right) u_{x}\left(x, y_{\ell+1 / 2}\right) d x\left(\delta_{x} \bar{v}_{H}\right)_{j, \ell+1 / 2} .
\end{aligned}
$$

The functional $\lambda(g):=g\left(\frac{1}{2}\right)-\int_{0}^{1} g(\xi) d \xi$ is bounded on $W^{2,1}(0,1)$ and vanishes for $g=1$ and $\xi$. Thus the BrambleHilbert Lemma gives the existence of a positive constant $C$ such that

$$
|\lambda(g)| \leqslant C\left\|g^{\prime \prime}\right\|_{L^{1}(0,1)} \text {. }
$$

From the last estimate applied to $g=w$, where

$$
w(\xi):=a\left(x_{j}, y_{\ell}+\xi k_{\ell}\right) \int_{x_{j-1 / 2}}^{x_{j+1 / 2}} u_{x}\left(x, y_{\ell}+\xi k_{\ell}\right) d x, \quad \xi \in[0,1],
$$


follows

$$
S_{1}=S_{1}^{(1)}-\sum_{j=p_{\ell}}^{p_{\ell}+N_{\ell}} \int_{y_{\ell}}^{y_{\ell+1}} \int_{x_{j-1 / 2}}^{x_{j+1 / 2}} E_{j, \ell}\left(\delta_{x} \bar{v}_{H}\right)_{j, \ell+1 / 2},
$$

with

$$
\left|E_{j, \ell}\right| \leqslant C k_{\ell}^{2}\left|a\left(x_{j}, \cdot\right) \int_{x_{j-1 / 2}}^{x_{j+1 / 2}} u_{x}(x, \cdot) d x\right|_{W^{2,1}\left(\left(y_{\ell}, y_{\ell+1}\right)\right)} .
$$

Let

$$
S_{2}:=\sum_{j=p_{\ell}}^{p_{\ell}+N_{\ell}-1} h_{j} k_{\ell}\left(M_{H} R_{G_{H}}\left(a u_{x}\right)_{x}\right)_{j+1 / 2, \ell+1 / 2} \bar{v}_{j+1 / 2, \ell+1 / 2},
$$

which can be written in the form

$$
S_{2}=S_{2}^{(1)}+\sum_{j=p_{\ell}}^{p_{\ell}+N_{\ell}-1} F_{j, \ell} \bar{v}_{j+1 / 2, \ell+1 / 2}
$$

where

$$
\begin{aligned}
S_{2}^{(1)} & :=\sum_{j=p_{\ell}}^{p_{\ell}+N_{\ell}-1} \int_{y_{\ell}}^{y_{\ell+1}} \int_{x_{j}}^{x_{j+1}}\left(a u_{x}\right)_{x}(x, y) d x d y \bar{v}_{j+1 / 2, \ell+1 / 2}, \\
F_{j, \ell} & :=\left(M_{H} R_{G_{H}}\left(a u_{x}\right)_{x}\right)_{j+1 / 2, \ell+1 / 2}-\int_{y_{\ell}}^{y_{\ell+1}} \int_{x_{j}}^{x_{j+1}}\left(a u_{x}\right)_{x}(x, y) d x d y .
\end{aligned}
$$

$F_{j, \ell}$ can be bounded with the aid of the Bramble-Hilbert Lemma. Let the function $w$ be defined by

$$
w(\xi, \eta):=\left(a u_{x}\right)_{x}\left(x_{j}+\xi h_{j}, y_{\ell}+\eta k_{\ell}\right), \quad(\xi, \eta) \in(0,1) \times(0,1) .
$$

Then

$$
F_{j, \ell}=h_{j} k_{\ell}\left(\frac{w(0,0)+w(1,0)+w(0,1)+w(1,1)}{4}-\int_{0}^{1} \int_{0}^{1} w(\xi, \eta) d \xi d \eta\right) .
$$

The functional

$$
\lambda(g):=\frac{g(0,0)+g(1,0)+g(0,1)+g(1,1)}{4}-\int_{0}^{1} \int_{0}^{1} g(\xi, \eta) d \xi d \eta,
$$

$g \in W^{2,2}((0,1) \times(0,1))$, is bounded and vanishes for $g=1, \xi$ and $\eta$. Again, by Bramble-Hilbert Lemma the estimate

$$
|\lambda(g)| \leqslant C|g|_{W^{2,1}((0,1) \times(0,1))}
$$

holds and we obtain the bound

$$
\begin{aligned}
\left|F_{j, \ell}\right| \leqslant & C\left(h_{j}^{2}\left\|\left(a u_{x}\right)_{x x x}\right\|_{L^{1}\left(\left(x_{j}, x_{j+1}\right) \times\left(y_{\ell}, y_{\ell+1}\right)\right)}+k_{\ell} h_{j}\left\|\left(a u_{x}\right)_{x x y}\right\|_{L^{1}\left(\left(x_{j}, x_{j+1}\right) \times\left(y_{\ell}, y_{\ell+1}\right)\right)}\right. \\
& \left.+k_{\ell}^{2}\left\|\left(a u_{x}\right)_{x y y}\right\|_{L^{1}\left(\left(x_{j}, x_{j+1}\right) \times\left(y_{\ell}, y_{\ell+1}\right)\right)}\right) .
\end{aligned}
$$

Let us finally consider the difference $S_{1}^{(1)}-S_{2}^{(1)}$. For $S_{2}^{(1)}$ we have 


$$
\begin{aligned}
S_{2}^{(1)} & =\sum_{j=p_{\ell}}^{p_{\ell}+N_{\ell}-1} \int_{y_{\ell}}^{y_{\ell+1}}\left(\left(a u_{x}\right)\left(x_{j+1}, y\right)-\left(a u_{x}\right)\left(x_{j}, y\right)\right) d y \bar{v}_{j+1 / 2, \ell+1 / 2} \\
& =-\sum_{j=p_{\ell}}^{p_{\ell}+N_{\ell}} \int_{y_{\ell}}^{y_{\ell+1}} \int_{x_{j-1 / 2}}^{x_{j+1 / 2}}\left(a u_{x}\right)\left(x_{j}, y\right) d x d y\left(\delta_{x} \bar{v}_{H}\right)_{j, \ell+1 / 2}
\end{aligned}
$$

and then

$$
S_{1}^{(1)}-S_{2}^{(1)}=\left(T_{1}+T_{2}\right) / 2+T_{3}+T_{4},
$$

with

$$
\begin{aligned}
& T_{1}:=-\sum_{j=p_{\ell}+1}^{p_{\ell}+N_{\ell}} \int_{y_{\ell}}^{y_{\ell}+1}\left[\frac{h_{j-1}}{2}\left(u_{x}\left(x_{j-1}, y\right)+u_{x}\left(x_{j}, y\right)\right)-\int_{x_{j-1}}^{x_{j}} u_{x}(x, y) d x\right] \\
& \times\left(a\left(x_{j-1}, y\right)\left(\delta_{x} \bar{v}_{H}\right)_{j-1, \ell+1 / 2}+a\left(x_{j}, y\right)\left(\delta_{x} \bar{v}_{H}\right)_{j, \ell+1 / 2}\right) d y, \\
& T_{2}:=-\sum_{j=p_{\ell}+1}^{p_{\ell}+N_{\ell}} \int_{y_{\ell}}^{y_{\ell+1}}\left[\frac{h_{j-1}}{2}\left(u_{x}\left(x_{j}, y\right)-u_{x}\left(x_{j-1}, y\right)\right)\right. \\
& \left.+\int_{x_{j-1}}^{x_{j-1 / 2}} u_{x}(x, y) d x-\int_{x_{j-1 / 2}}^{x_{j}} u_{x}(x, y) d x\right]\left(a\left(x_{j}, y\right)\left(\delta_{x} \bar{v}_{H}\right)_{j, \ell+1 / 2}-a\left(x_{j-1}, y\right)\left(\delta_{x} \bar{v}_{H}\right)_{j-1, \ell+1 / 2}\right) d y, \\
& T_{3}:=-\int_{y_{\ell}}^{y_{\ell}+1}\left[\frac{h_{p_{\ell}-1}}{2} u_{x}\left(x_{p_{\ell}}, y\right)-\int_{x_{p_{\ell}-1 / 2}}^{x_{p_{\ell}}} u_{x}(x, y) d x\right] a\left(x_{p_{\ell}}, y\right) d y\left(\delta_{x} \bar{v}_{H}\right)_{p_{\ell}, \ell+1 / 2}, \\
& T_{4}:=-\int_{y_{\ell}}^{y_{\ell+1}}\left[\frac{h_{p_{\ell}+N_{\ell}}}{2} u_{x}\left(x_{p_{\ell}+N_{\ell}}, y\right)-\int_{x_{p_{\ell}+N_{\ell}}}^{x_{p_{\ell}+N_{\ell}+1 / 2}} u_{x}(x, y) d x\right] a\left(x_{p_{\ell}+N_{\ell}}, y\right) d y\left(\delta_{x} \bar{v}_{H}\right)_{p_{\ell}+N_{\ell}, \ell+1 / 2} .
\end{aligned}
$$

The sum in $T_{1}$ contains the errors of the trapezoidal rule that can be bounded with the aid of the Bramble-Hilbert Lemma by

$$
\begin{aligned}
\left|T_{1}\right| \leqslant & C \sum_{j=p_{\ell}+1}^{p_{\ell}+N_{\ell}} h_{j-1}^{2}\left\|u_{x x x}\right\|_{L^{1}\left(\left(x_{j}, x_{j+1}\right) \times\left(y_{\ell}, y_{\ell+1}\right)\right)}\|a\|_{L^{\infty}\left(\left(x_{j}, x_{j+1}\right) \times\left(y_{\ell}, y_{\ell+1}\right)\right)} \\
& \times\left(\left|\left(\delta_{x} \bar{v}_{H}\right)_{j-1, \ell+1 / 2}\right|+\left|\left(\delta_{x} \bar{v}_{H}\right)_{j, \ell+1 / 2}\right|\right) .
\end{aligned}
$$

For $T_{2}$ we have only the first order bound but the factor

$$
a\left(x_{j}, y\right)\left(\delta_{x} \bar{v}_{H}\right)_{j, \ell+1 / 2}-a\left(x_{j-1}, y\right)\left(\delta_{x} \bar{v}_{H}\right)_{j-1, \ell+1 / 2}
$$

allows to estimate $T_{2}$ with the same order as $T_{1}$. We have

$$
\begin{aligned}
& a\left(x_{j}, y\right)\left(\delta_{x} \bar{v}_{H}\right)_{j, \ell+1 / 2}-a\left(x_{j-1}, y\right)\left(\delta_{x} \bar{v}_{H}\right)_{j-1, \ell+1 / 2} \\
& =a\left(x_{j-1 / 2}, y\right)\left(\left(\delta_{x} \bar{v}_{H}\right)_{j, \ell+1 / 2}-\left(\delta_{x} \bar{v}_{H}\right)_{j-1, \ell+1 / 2}\right)+\left(a\left(x_{j-1 / 2}, y\right)-a\left(x_{j-1}, y\right)\right)\left(\delta_{x} \bar{v}_{H}\right)_{j-1, \ell+1 / 2} \\
& \quad+\left(a\left(x_{j}, y\right)-a\left(x_{j-1 / 2}, y\right)\right)\left(\delta_{x} \bar{v}_{H}\right)_{j, \ell+1 / 2} \\
& =h_{j-1} a\left(x_{j-1 / 2}, y\right)\left(\delta_{x}^{2} \bar{v}_{H}\right)_{j-1 / 2, \ell+1 / 2}+\frac{h_{j-1}}{2}\left(a_{x}\left(\eta_{1}, y\right)\left(\delta_{x} \bar{v}_{H}\right)_{j-1, \ell+1 / 2}+a_{x}\left(\eta_{2}, y\right)\left(\delta_{x} \bar{v}_{H}\right)_{j, \ell+1 / 2}\right),
\end{aligned}
$$

for some $\eta_{1} \in\left[x_{j-1}, x_{j-1 / 2}\right], \eta_{2} \in\left[x_{j-1 / 2}, x_{j}\right]$, and then 


$$
\begin{aligned}
\left|T_{2}\right| \leqslant & C \sum_{j=p_{\ell}+1}^{p_{\ell}+N_{\ell}} h_{j-1}^{2}\left\|u_{x x}\right\|_{L^{1}\left(\left(x_{j}, x_{j+1}\right) \times\left(y_{\ell}, y_{\ell+1}\right)\right)}\|a\|_{W^{1, \infty}\left(\left(x_{j}, x_{j+1}\right) \times\left(y_{\ell}, y_{\ell+1}\right)\right)} \\
& \times\left(\left|\left(\delta_{x}^{2} \bar{v}_{H}\right)_{j-1 / 2, \ell+1 / 2}\right|+\left|\left(\delta_{x} \bar{v}_{H}\right)_{j-1, \ell+1 / 2}\right|+\left|\left(\delta_{x} \bar{v}_{H}\right)_{j, \ell+1 / 2}\right|\right) .
\end{aligned}
$$

For $T_{3}$ and $T_{4}$ we have

$$
\begin{aligned}
& \left|T_{3}\right| \leqslant \int_{y_{\ell}}^{y_{\ell+1}} \frac{h_{p_{\ell}-1}}{8}\left\|u_{x x}(\cdot, y)\right\|_{L^{1}\left(\left(x_{p_{\ell}-1 / 2}, x_{p_{\ell}}\right)\right)}\left|a\left(x_{p_{\ell}}, y\right)\right| d y\left|\left(\delta_{x} \bar{v}_{H}\right)_{p_{\ell}, \ell+1 / 2}\right|, \\
& \left|T_{4}\right| \leqslant \int_{y_{\ell}}^{y_{\ell+1}} \frac{h_{p_{\ell}+N_{\ell}}}{8}\left\|u_{x x}(\cdot, y)\right\|_{L^{1}\left(\left(x_{p_{\ell}+N_{\ell}}, x_{\left.p_{\ell}+N_{\ell}+1 / 2\right)}\right)\right.}\left|a\left(x_{p_{\ell}+N_{\ell}}, y\right)\right| d y\left|\left(\delta_{x} \bar{v}_{H}\right)_{p_{\ell}+N_{\ell}, \ell+1 / 2}\right| .
\end{aligned}
$$

Considering the equality

$$
\left(\delta_{x} \bar{v}_{H}\right)_{p_{\ell}, \ell+1 / 2}=-\sum_{i=p_{\ell}}^{j} h_{i}\left(\delta_{x}^{2} \bar{v}_{H}\right)_{i+1 / 2, \ell+1 / 2}+\left(\delta_{x} \bar{v}_{H}\right)_{j+1, \ell+1 / 2}
$$

$j=p_{\ell}, \ldots, p_{\ell}+N_{\ell}-1$, follows

$$
\left|\left(\delta_{x} \bar{v}_{H}\right)_{p_{\ell}, \ell+1 / 2}\right| \leqslant \sum_{j=p_{\ell}}^{p_{\ell}+N_{\ell}-1} h_{j}\left|\left(\delta_{x}^{2} \bar{v}_{H}\right)_{j+1 / 2, \ell+1 / 2}\right|+\frac{1}{x_{p_{\ell}+N_{\ell}}-x_{p_{\ell}}} \sum_{j=p_{\ell}}^{p_{\ell}+N_{\ell}-1} h_{j}\left|\left(\delta_{x} \bar{v}_{H}\right)_{j+1, \ell+1 / 2}\right| .
$$

For $T_{3}$ we have

$$
\begin{aligned}
\left|T_{3}\right| \leqslant & \frac{h_{p_{\ell}-1}}{8}\left\|u_{x x}\right\|_{L^{1}\left(\left(x_{p_{\ell}-1 / 2}, x_{p_{\ell}}\right) \times\left(y_{\ell}, y_{\ell+1}\right)\right)}\left\|a\left(x_{p_{\ell}}, \cdot\right)\right\|_{L^{\infty}\left(\left(y_{\ell}, y_{\ell+1}\right)\right)} \\
& \times\left(\sum_{j=p_{\ell}}^{p_{\ell}+N_{\ell}-1} h_{j}\left|\left(\delta_{x}^{2} \bar{v}_{H}\right)_{j+1 / 2, \ell+1 / 2}\right|+\frac{1}{x_{p_{\ell}+N_{\ell}}-x_{p_{\ell}}} \sum_{j=p_{\ell}}^{p_{\ell}+N_{\ell}-1} h_{j}\left|\left(\delta_{x} \bar{v}_{H}\right)_{j+1, \ell+1 / 2}\right|\right),
\end{aligned}
$$

and in the same way for $T_{4}$ we obtain

$$
\begin{aligned}
\left|T_{4}\right| \leqslant & \frac{h_{p_{\ell}+N_{\ell}}}{8}\left\|u_{x x}\right\|_{L^{1}\left(\left(x_{p_{\ell}+N_{\ell}}, x_{p_{\ell}+N_{\ell}+1 / 2}\right) \times\left(y_{\ell}, y_{\ell+1}\right)\right)}\left\|a\left(x_{p_{\ell}+N_{\ell}}, \cdot\right)\right\|_{L^{\infty}\left(\left(y_{\ell}, y_{\ell+1}\right)\right)} \\
& \times\left(\sum_{j=p_{\ell}}^{p_{\ell}+N_{\ell}-1} h_{j}\left|\left(\delta_{x}^{2} \bar{v}_{H}\right)_{j+1 / 2, \ell+1 / 2}\right|+\frac{1}{x_{p_{\ell}+N_{\ell}}-x_{p_{\ell}}} \sum_{j=p_{\ell}}^{p_{\ell}+N_{\ell}-1} h_{j}\left|\left(\delta_{x} \bar{v}_{H}\right)_{j+1, \ell+1 / 2}\right|\right) .
\end{aligned}
$$

Using the Schwarz's inequality we obtain (24).

Lemma 9. Let $u \in H^{3}(\Omega)$. Then the following estimates hold

$$
\begin{aligned}
& \left|\left(M_{x}\left(d \delta_{x} u\right), v_{H}\right)_{H}-\left(M_{H} R_{G_{H}}\left(d u_{x}\right), v_{H}\right)_{H}\right| \\
& \quad \leqslant C\|d\|_{W^{2, \infty}(\Omega)}\left(\sum_{\Omega_{H}}\left(h_{j}^{2}+k_{\ell}^{2}\right)^{2}\left\|u_{x x x}\right\|_{L^{2}\left(\left(x_{j}, x_{j+1}\right) \times\left(y_{\ell}, y_{\ell+1}\right)\right)}^{2}\right)^{1 / 2}\left\|v_{H}\right\|_{1, H}, \\
& \left|\left(M_{y}\left(e \delta_{y} u\right), v_{H}\right)_{H}-\left(M_{H} R_{G_{H}}\left(e u_{y}\right), v_{H}\right)_{H}\right| \\
& \quad \leqslant C\|e\|_{W^{2, \infty}(\Omega)}\left(\sum_{\Omega_{H}}\left(h_{j}^{2}+k_{\ell}^{2}\right)^{2}\left\|u_{y y y}\right\|_{L^{2}\left(\left(x_{j}, x_{j+1}\right) \times\left(y_{\ell}, y_{\ell+1}\right)\right)}^{2}\right)^{1 / 2}\left\|v_{H}\right\|_{1, H},
\end{aligned}
$$

for all $v_{H} \in \stackrel{\circ}{W}_{H}^{1,2}(\Omega)$. 
Proof. Let us consider the terms in $\left(M_{x}\left(d \delta_{x} u\right), v_{H}\right)_{H}$ and $\left(M_{H} R_{G_{H}}\left(d u_{x}\right), v_{H}\right)_{H}$ which have the factor $\bar{v}_{j+1 / 2, \ell+1 / 2}$, for some $j$, with $\ell$ given. We obtain for $\left(M_{x}\left(d \delta_{x} u\right), v_{H}\right)_{H}$ and $\left(M_{H} R_{G_{H}}\left(d u_{x}\right), v_{H}\right)_{H}$, respectively,

$$
\begin{aligned}
& \sum_{j=p_{\ell}}^{p_{\ell}+N_{\ell}-1} k_{\ell} h_{j}\left(M_{x}\left(d \delta_{x} u\right)\right)_{j+1 / 2, \ell+1 / 2} \bar{v}_{j+1 / 2, \ell+1 / 2} \\
& =-\sum_{j=p_{\ell}}^{p_{\ell}+N_{\ell}} k_{\ell} h_{j-1 / 2} \sum_{i=p_{\ell}}^{j-1} h_{i}\left(M_{x}\left(d \delta_{x} u\right)\right)_{i+1 / 2, \ell+1 / 2}\left(\delta_{x} \bar{v}_{H}\right)_{j, \ell+1 / 2}
\end{aligned}
$$

and

$$
\begin{aligned}
& \sum_{j=p_{\ell}}^{p_{\ell}+N_{\ell}-1} k_{\ell} h_{j}\left(M_{H} R_{G_{H}}\left(d u_{x}\right)\right)_{j+1 / 2, \ell+1 / 2} \bar{v}_{j+1 / 2, \ell+1 / 2} \\
& =-\sum_{j=p_{\ell}}^{p_{\ell}+N_{\ell}} k_{\ell} h_{j-1 / 2} \sum_{i=p_{\ell}}^{j-1} h_{i}\left(M_{x}\left(d u_{x}\right)\right)_{i+1 / 2, \ell+1 / 2}\left(\delta_{x} \bar{v}_{H}\right)_{j, \ell+1 / 2} \\
& \quad+\sum_{j=p_{\ell}}^{p_{\ell}+N_{\ell}-1} \frac{h_{j}}{2} k_{\ell}\left(\left(E_{y}\right)_{j, \ell+1 / 2}+\left(E_{y}\right)_{j+1, \ell+1 / 2}\right) \bar{v}_{j+1 / 2, \ell+1 / 2},
\end{aligned}
$$

where

$$
\left(E_{y}\right)_{j, \ell+1 / 2}:=\frac{\left(d u_{x}\right)_{j, \ell}+\left(d u_{x}\right)_{j, \ell+1}}{2}-\left(d u_{x}\right)_{j, \ell+1 / 2} .
$$

Let $w(\xi):=\left(d u_{x}\right)\left(x_{j}, y_{\ell}+\xi k_{\ell}\right), \xi \in[0,1]$. Then

$$
\left(E_{y}\right)_{j, \ell+1 / 2}=\frac{w(0)+w(1)}{2}-w\left(\frac{1}{2}\right) .
$$

The functional $\lambda(g):=\frac{g(0)+g(1)}{2}-g\left(\frac{1}{2}\right)$ is bounded on $W^{2,1}(0,1)$ and vanishes for $g=1$ and $\xi$. Again by the Bramble-Hilbert Lemma the estimate

$$
|\lambda(g)| \leqslant C\left\|g^{\prime \prime}\right\|_{L^{1}(0,1)}, \quad g \in W^{2,1}(0,1),
$$

holds and we obtain the bound

$$
\begin{aligned}
& \sum_{j=p_{\ell}}^{p_{\ell}+N_{\ell}-1} \frac{h_{j}}{2} k_{\ell}\left|\left(E_{y}\right)_{j, \ell+1 / 2}+\left(E_{y}\right)_{j+1, \ell+1 / 2}\right|\left|v_{j+1 / 2, \ell+1 / 2}\right| \\
& \leqslant \sum_{j=p_{\ell}}^{p_{\ell}+N_{\ell}-1} \frac{h_{j}}{2} k_{\ell}^{2}\left(\left\|\left(\left(d u_{x}\right)_{x x}\right)\left(x_{j}, \cdot\right)\right\|_{L^{1}\left(I_{\ell}\right)}+\left\|\left(\left(d u_{x}\right)_{x x}\right)\left(x_{j+1}, \cdot\right)\right\|_{L^{1}\left(I_{\ell}\right)}\right)\left|v_{j+1 / 2, \ell+1 / 2}\right| .
\end{aligned}
$$

We have

$$
\begin{aligned}
& \sum_{i=p_{\ell}}^{j-1} h_{i}\left[\left(M_{x}\left(d \delta_{x} u\right)\right)_{i+1 / 2, \ell+1 / 2}-\left(M_{x}\left(d u_{x}\right)\right)_{i+1 / 2, \ell+1 / 2}\right] \\
& =\sum_{i=p_{\ell}+1}^{j-1} h_{i-1 / 2} d_{i, \ell+1 / 2}\left(\left(\delta_{x} u\right)_{i, \ell+1 / 2}-u_{x}\left(x_{i}, y_{\ell+1 / 2}\right)\right)+\frac{h_{j-1}}{2} d_{j, \ell+1 / 2}\left(\left(\delta_{x} u\right)_{j, \ell+1 / 2}-u_{x}\left(x_{j}, y_{\ell+1 / 2}\right)\right) \\
& \quad+\frac{h_{p_{\ell}}}{2} d_{p_{\ell}, \ell+1 / 2}\left(\left(\delta_{x} u\right)_{p_{\ell}, \ell+1 / 2}-u_{x}\left(x_{p_{\ell}}, y_{\ell+1 / 2}\right)\right) .
\end{aligned}
$$

Using (28) we obtain the bound (26). 
Lemma 10. Let $w \in H^{2}(\Omega)$. Then, for all $v_{H} \in \stackrel{\circ}{L}_{H}^{2}(\Omega)$,

$$
\left|\left(R_{H} w, v_{H}\right)_{H}-\left(M_{H} R_{G_{H}} w, v_{H}\right)_{H}\right| \leqslant C\left(\sum_{\Omega_{H}}\left(h_{j}^{2}+k_{\ell}^{2}\right)^{2}\|w\|_{H^{2}\left(\left(x_{j}, x_{j+1}\right) \times\left(y_{\ell}, y_{\ell+1}\right)\right)}^{2}\right)^{1 / 2}\left\|v_{H}\right\|_{0, H} .
$$

Proof. We can write

$$
\left(M_{H} R_{G_{H}} w\right)_{j+1 / 2, \ell+1 / 2}=w_{j+1 / 2, \ell+1 / 2}+\left(E_{x}\right)_{j+1 / 2, \ell}+\left(E_{x}\right)_{j+1 / 2, \ell+1}+\left(E_{y}\right)_{j+1 / 2, \ell+1 / 2},
$$

where

$$
\begin{aligned}
& \left(E_{x}\right)_{j+1 / 2, \ell}:=\frac{w_{j, \ell}+w_{j+1, \ell}}{4}-\frac{w_{j+1 / 2, \ell}}{2}, \\
& \left(E_{y}\right)_{j+1 / 2, \ell+1 / 2}:=\frac{w_{j+1 / 2, \ell}+w_{j+1 / 2, \ell+1}}{2}-w_{j+1 / 2, \ell+1 / 2} .
\end{aligned}
$$

Using the Bramble-Hilbert Lemma as before we obtain (29).

Let us consider in (29) $w=f u$. For all $v_{H} \in \stackrel{\circ}{L}_{H}^{2}(\Omega)$ we obtain

$$
\begin{aligned}
& \left|\left(f u, v_{H}\right)_{H}-\left(M_{H} R_{G_{H}}(f u), v_{H}\right)_{H}\right| \\
& \quad \leqslant C\|f\|_{W^{2, \infty}(\Omega)} H_{\max }^{2}\left(\sum_{\Omega_{H}}\|u\|_{H^{2}\left(\left(x_{j}, x_{j+1}\right) \times\left(y_{\ell}, y_{\ell+1}\right)\right)}^{2}\right)^{1 / 2}\left\|v_{H}\right\|_{0, H} .
\end{aligned}
$$

The next result follows from Theorem 2 and from the bounds (24)-(27) and (30).

Theorem 3. Let $\Omega$ be a union of rectangles. Assume that the solution u of (1)-(2) lies in $H^{4}(\Omega)$. Then for $H \in \Lambda$, with $H_{\max }$ small enough, the discrete problem (3)-(4) has a unique solution $u_{H}$ which satisfies

$$
\begin{aligned}
\left\|R_{H} u-u_{H}\right\|_{0, H} & \leqslant C\left(\sum_{\Omega_{H}}\left(h_{j}^{2}+k_{\ell}^{2}\right)^{2}\|u\|_{H^{4}\left(\left(x_{j}, x_{j+1}\right) \times\left(y_{\ell}, y_{\ell+1}\right)\right)}^{2}\right)^{1 / 2} \\
& \leqslant C H_{\max }^{2}\|u\|_{H^{4}(\Omega)} .
\end{aligned}
$$

\section{Numerical results}

We present numerical results for the problem

$$
\begin{aligned}
& -\Delta u=f \quad \text { on } \Omega=(0,1) \times(0,1), \\
& u=0 \quad \text { on } \partial \Omega,
\end{aligned}
$$

with $f(x, y)=-\left((x(x-1) y(y-1))^{2}\right)_{x x}-\left((x(x-1) y(y-1))^{2}\right)_{y y}$. Fig. 2 shows the numerical solution on 500 random meshes ( $N-1 \times M-1$ points placed in $\Omega$ at random), where $N$ and $M$ ranges from 10 to 110 . The logarithm of the norm of the error, $\log \left(\left\|R_{H} u-u_{H}\right\|_{0, H}\right)$, is plotted versus the logarithm of the maximum step-size. The straight line is the least-squares fit to the points and has the slope 2.1721, which confirms the estimates given in Theorem 3.

\section{Acknowledgements}

The research work compiled in the present paper significantly benefited from the suggestions of Rolf Dieter Grigorieff and José Augusto Ferreira to whom the author wishes to express sincere gratitude. The author also thanks the helpful suggestions of the referees. The author gratefully acknowledge the support of this work by the Centro de Matemática da Universidade de Coimbra and Fundação para a Ciência e Tecnologia. 


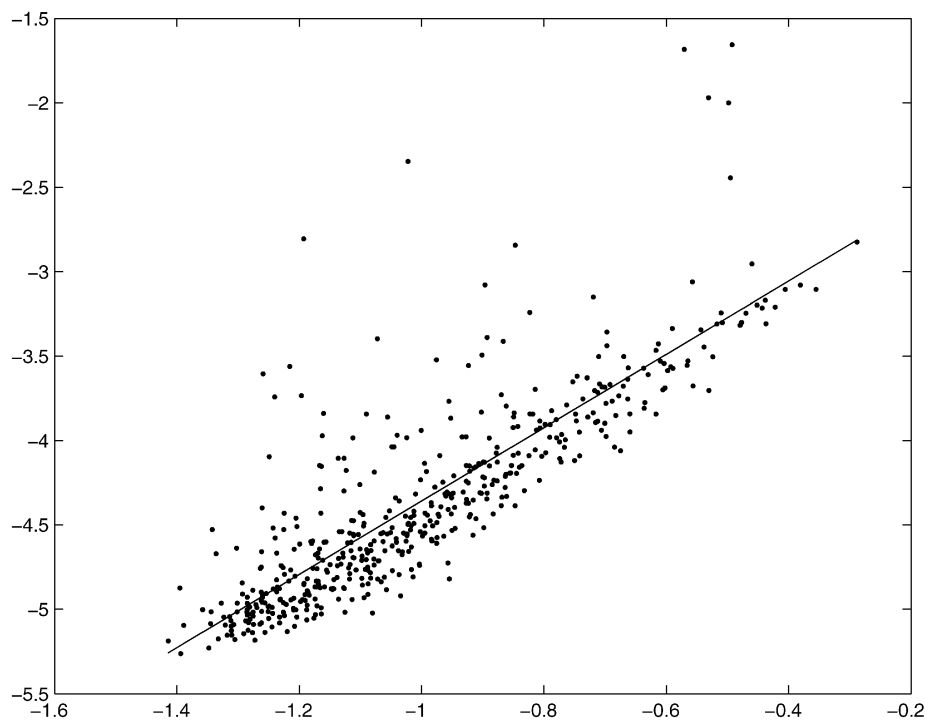

Fig. 2. $\log \left(\left\|R_{H} u-u_{H}\right\|_{0, H}\right)$ versus $\log \left(h_{\max }\right)$.

\section{References}

[1] S. Barbeiro, Discretely compact imbeddings in spaces of cell-centered grid functions, Pré-Publicações do Departamento de Matemática, Universidade de Coimbra, 06-46, 2006.

[2] S. Barbeiro, Normas Duais Discretas em Problemas Elípticos, Ph.D. Thesis, Universidade de Coimbra, 2005.

[3] S. Barbeiro, J.A. Ferreira, R.D. Grigorieff, Supraconvergence of a finite difference scheme for solutions in $H^{s}(0, L)$, IMA J. Numer. Anal. 25 (2005) 797-811.

[4] D. Bojović, B.S. Jovanović, Fractional order convergence rate estimates of finite difference method on nonuniform meshes, Comput. Methods Appl. Math. 1 (2001) 213-221.

[5] J.H. Bramble, S.R. Hilbert, Bounds for a class of linear functionals with applications to Hermite interpolation, Numer. Math. 16 (1970/1971) 362-369.

[6] F. de Hoog, D. Jackett, On the rate of convergence of finite difference schemes on nonuniform grids, J. Austral. Math. Soc. Ser. B 26 (1985) 247-256.

[7] J.A. Ferreira, R.D. Grigorieff, Supraconvergence and supercloseness of a scheme for elliptic equations on non-uniform grids, Numer. Funct. Anal. Optim. 27 (5-6) (2006) 539-564.

[8] J.A. Ferreira, Supraconvergence of elliptic finite difference schemes: general boundary conditions and low regularity, Pré-publicações do Departamento de Matemática da Universidade de Coimbra 04-27, 2004.

[9] J.A. Ferreira, On the convergence on nonrectangular grids, J. Comp. Appl. Math. 85 (1997) 333-344.

[10] J.A. Ferreira, R.D. Grigorieff, On the supraconvergence of elliptic finite difference schemes, Appl. Numer. Math. 28 (1998) $275-292$.

[11] P.A. Forsyth, P.H. Sammon, Quadratic convergence for cell-centered grids, Appl. Numer. Math. 4 (1988) 377-394.

[12] B. Garcia-Archila, A supraconvergent scheme for the Korteweg-de Vries equation, Numer. Math. 61 (1992) $292-310$.

[13] G. Goodsell, J.R. Whiteman, A unified treatment of superconvergent recovered gradient functions for piecewise linear finite element approximations, Internat. J. Numer. Methods Engrg. 27 (1989) 469-481.

[14] R.D. Grigorieff, Diskret kompakte Einbettungen in Sobolewschen Räumen, Math. Ann. 197 (1972) 71-85.

[15] R.D. Grigorieff, Some stability inequalities for compact finite difference operators, Math. Nachr. 135 (1988) 93-101.

[16] B.S. Jovanović, L.D. Ivanović, E.E. Süli, Convergence of finite difference schemes for elliptic equations with variable coefficients, IMA J. Numer. Anal. 7 (1987) 301-305.

[17] B.S. Jovanović, B.Z. Popović, Convergence of a finite difference scheme for the third boundary-value problem for an elliptic equation with variable coefficients, Comput. Methods Appl. Math. 1 (2001) 356-366.

[18] H.O. Kreiss, T.A. Manteuffel, B. Swartz, B. Wendroff, A.B. White Jr, Supraconvergent schemes on irregular grids, Math. Comp. 47 (1986) 537-554.

[19] R. Lazarov, V. Makarov, W. Weinelt, On the convergence of difference schemes for the approximation of solutions $u \in W_{2}^{m}(m>0.5)$ of elliptic equations with mixed derivatives, Numer. Math. 44 (1984) 223-232.

[20] P. Lesaint, M. Zlámal, Superconvergence of the gradient of finite element solutions, R.A.I.R.O. Analyse Numérique 13 (1979) $139-166$.

[21] C.D. Levermore, T.A. Manteuffel, A.B. White Jr., Numerical solutions of partial differential equations on irregular grids, in: Computational Techniques and Applications, CTAC-87, North-Holland, Amsterdam, 1987, pp. 417-426.

[22] N. Levine, Superconvergent recovery of the gradient from piecewise linear finite element approximations, IMA J. Numer. Anal. 5 (1985) 407-427. 
[23] Q. Lin, L. Tobiska, A. Zhou, Superconvergence and extrapolation of non-conforming low order finite elements applied to the Poisson equation, IMA J. Numer. Anal. 25 (1) (2005) 160-181.

[24] T.A. Manteuffel, A.B. White Jr., The numerical solution of second order boundary value problems on nonuniform meshes, Math. Comp. 47 (1986) 511-535.

[25] L.A. Oganesjan, L.A. Ruhovec, An investigation of the rate of convergence of variation-difference schemes for second order elliptic equations in a two-dimensional region with smooth boundary, Ž. Vyčisl. Mat. i Mat. Fiz. 9 (1969) 1102-1120.

[26] T.F. Russell, M.F. Wheeler, Finite element and finite difference methods for continuous flows in porous media, in: R.E. Ewing (Ed.), The Mathematics of Reservoir Simulation, SIAM, Philadelphia, 1983, pp. 35-106.

[27] A.A. Samarskii, Local one dimensional schemes on nonuniform nets, USSR Comput. Math. Math. Phys. 3 (1963) $572-619$.

[28] M.N. Spijker, Stability and convergence of finite-difference methods, Thesis, Leiden, 1968.

[29] F. Stummel, Diskrete Konvergenz linearer Operatoren. I, Math. Ann. 190 (1970) 45-92.

[30] F. Stummel, Discrete convergence of mappings, in: Topics in Numerical Analysis, Academic Press, London, 1972, pp. $285-310$.

[31] A.N. Tikhonov, A.A. Samarskii, Homogeneous difference schemes on nonuniform nets, USSR Comput. Math. Math. Phys. 2 (1962) 927-953.

[32] A. Weiser, M.F. Wheeler, On convergence of block-centered finite differences for elliptic problems, SIAM J. Numer. Anal. 25 (1988) 351-375.

[33] M. Zlámal, Superconvergence and reduced integration in finite element method, Math. Comp. 32 (1978) $663-685$. 